\title{
Publisher Notice
}

DOI 10.1515/scid-2014-5001

De Gruyter is happy to announce new editorial leadership for Statistical Communications in Infectious Diseases (SCID). We welcome Dr. Scott Evans, Department of Biostatistics and the Center for Biostatistics in AIDS Research, Harvard University, as Editor-in-Chief. Dr. Evans has put together a diverse editorial team who together aim to publish significant research on the application of statistical ideas to problems arising from studies of infectious diseases. SCID takes a broad perspective on the role of statistics in infectious disease research including application, policy, education and theory. SCID fosters much-needed communication among statisticians on the best approaches to evolving complex infectious disease data, and is a venue for statisticians to enter a dialogue with other scientists and policy makers on the strengths and limitations of policies and methods for design, monitoring, analysis, and reporting of infectious disease research studies.

SCID will resume regular publication as of January 2017.

The De Gruyter Publications Team 